\title{
TEFROESTRATIGRAFÍA (40 000-2000 a.P.) EN EL SECTOR CARIBE DE LOS VOLCANES BARVA, CONGO Y HULE, CORDILLERA CENTRAL, COSTA RICA
}

\author{
Guillermo E. Alvarado ${ }^{1} \&$ Flavia M. Salani ${ }^{2}$
}

\author{
${ }^{1}$ Área de Amenazas y Auscultación Sísmica y Volcánica, Instituto Costarricense de \\ Electricidad, Apdo. 10032-1000, San José, Costa Rica \\ Autor para correspondencia: galvaradoi@ice.go.cr \\ ${ }^{2}$ Departamento de Ciencias Geológicas, Universidad de Buenos Aires, Pabellón II, \\ Ciudad Universitaria, C-1428 EHA, Buenos Aires, Argentina
}

(Recibido 28/03/03; aceptado 08/08/03)

\begin{abstract}
The area is located in the north side of the Central Volcanic Range, near the Poás (historically active), Von Frantzius (active at least 40000 years B.P.) and Congo (active about 6000 years B.P.) volcanoes. It comprises the Hule maar that exploded 2800 years ago and possibly showed some minor eruptive periods about 1700 and 670 years B.P., and the Barva volcano. Pumice sheets (airfall and flow) which crop out in both margins of the Sarapiquí river are older than 40000 years. Field survey showed the existence of thick pyroclastic sheets (phreatomagmatic tuffs and plinian lapilli) from the Congo and Hule volcanoes. Among the facies associated with Congo we identified airfall facies of andesitic composition and corresponding to different eruptions $\left(\mathrm{C}_{1}\right.$ y $\left.\mathrm{C}_{2}\right)$; pumice and -less frequent- block and ash pyroclastic flow facies and a lahar facies with a radiocarbon age of 6030 years. Within the explosive event related with the Hule volcanic center, besides the plinian airfall facies, there are stratified tuffs and lapilli deposits corresponding to base surges and ash cloud surges.
\end{abstract}

Keywords: Tephrastratigraphy, Barva volcano, Congo volcano, Hule volcano, plinian deposits, piroclastic surges, radiocarbon ages.

RESUMEN: El área de estudio se ubica en el flanco norte de la Cordillera Volcánica Central, en las inmediaciones de los volcanes Poás (históricamente activo), Von Frantizius (activo por lo menos hace 40000 años), Congo (activo hace unos 6000 años) y comprende la caldera explosiva de la Laguna Hule, que explotó hace 2800 años posiblemente con periodos eruptivos menores hace 1700 y 670 años a.P. y el volcán Barva. Las capas de pómez plinianas (caída y flujos) aflorantes en ambas márgenes del río Sarapiquí son antiguas (> 40000 años). Se encontraron capas piroclásticas (tobas freatomagmáticas y lapilli pliniano) holocenas con fragmentos líticos y juveniles, procedentes de los volcanes Congo y Hule. Entre las facies asociadas con Congo, se identificaron facies de caída de naturaleza andesítica, correspondientes con distintas erupciones $\left(\mathrm{C}_{1}\right.$ y $\left.\mathrm{C}_{2}\right)$; facies de flujo piroclástico (pumíceos y menos frecuentemente de bloques y ceniza) y una facies lahárica (datada en 6030 años). Dentro del evento explosivo relacionado con el centro volcánico Hule, se encuentran además de la facies de caída pliniana, depósitos de tobas estratificadas y lapillitas vinculados con procesos de oleadas piroclásticas basales y oleadas asociadas con nubes de cenizas.

Palabras clave: Tefroestratigrafía, volcán Barva, volcán Congo, volcán Hule, depósitos plinianos, oleadas piroclásticas, edades de radiocarbono. 


\section{INTRODUCCIÓN}

Los volcanes Barva, Congo y Hule se ubican en la vertiente caribe de la Cordillera Volcánica Central de Costa Rica (Fig. 1) conformando parte de los macizos del Poás y Barva. Se alinean a lo largo de sistemas fisurales de orientación N$\mathrm{S}$, que corresponden con fracturas de debilidad cortical profunda. Por una parte se alinean en un sistema que reúne al Poás, Congo, maar Hule y maar Río Cuarto y por otra constituyen el sistema Barva-Cacho Negro. Los productos más comunes de estos centros son lavas, depósitos laháricos y rocas piroclásticas cuya edad es $<40000$
a.P. Las lavas más antiguas dentro del área estudiada corresponden con flujos emitidos por el volcán Von Frantzius (activo por lo menos hace 40000 años) y las erupciones explosivas con piroclastitas relacionadas con el volcán Barva, Congo (6000 años a.P) y con el maar de la laguna Hule (2800 años, posiblemente con periodos eruptivos menores).

En este trabajo se presenta una síntesis de la información geovulcanológica, se exponen nuevos datos obtenidos mediante el levantamiento de secciones en los depósitos de rocas piroclásticas, se aportan dataciones radiocarbónicas y, finalmente, los resultados que surgen del análisis


Fig. 1: Mapa de ubicación del área estudiada. 
tefro-cronoestratigráfico, complementados con mapas de isopacas y de isopletas.

El estudio fue realizado dentro del marco de la evaluación del peligro y riesgo volcánico para los proyectos hidroeléctricos Cariblanco y Toro III llevados a cabo por el Instituto Costarricense de Electricidad (Alvarado \& Salani, 2002).

\section{SÍNTESIS GEOVULCANOLÓGICA DE LOS MACIZOS DEL POÁS Y BARVA}

\section{Macizo del Poás}

El Poás es un complejo estratovolcánico activo cuya edificación debe de datar del Pleistoceno Medio. Su amplia cúspide está coronada por tres focos volcánicos principales orientados N-S: cono Botos, el cráter activo y el cono Von Frantzius. Estas estructuras se sobreponen a un macizo volcánico más antiguo (Paleo-Poás) y a su vez están controladas por la tectónica regional en dirección N-S, sobre la cual se emplazaron varios focos volcánicos menores (Fig. 2): conos piroclásticos de Sabana Redonda (al sur del cráter activo del Poás), volcán Congo, y los maares de Hule y Río Cuarto, todos ellos al norte del Poás.

Los estudios recientes en el Poás se han enfocado principalmente a su actividad histórica y a la comprensión del fenómeno en general, a su petrología y geología local, a la geoquímica de sus exhalaciones y de la salmuera cratérica o a aspectos particulares de la geofísica. Muy poco se ha referido a su contexto geoestructural y mucho menos a su tefraestratigrafía. Una información geovulcanológica detallada de parte del macizo del Poás y del Barva, así como una revisión de los trabajos anteriores, se exponen en su mayoría en los trabajos de Soto (1999) y Alvarado \& Salani (2002).

\section{Volcán Congo}

El volcán Congo se localiza entre el volcán Von Frantzius y la Depresión de Bosque Alegre (Figs. 1 y 2). Es un cono bien desarrollado hacia el norte y menos hacia el sur, debido al obstáculo morfológico que le representaba parte del cono Von Frantzius. Es de moderado tamaño: altura 2014 m s.n.m.; se levanta unos $1300 \mathrm{~m}$ sobre la planicie norte y tiene un volumen de unos $3 \mathrm{~km}^{3}$ (Soto, 1999). El volcán Cerro Congo parece haberse desarrollado contemporánea o ligeramente anterior al cono Von Frantzius. Al menos las coladas de lava de éste último, que alcanzan el área más septentrional, fueron desviadas por la barrera morfológica del Congo. La parte superior del cono es un cráter abierto en dos herraduras erosivas hacia el NNO y al NNE, probablemente formadas posterior a su última erupción.

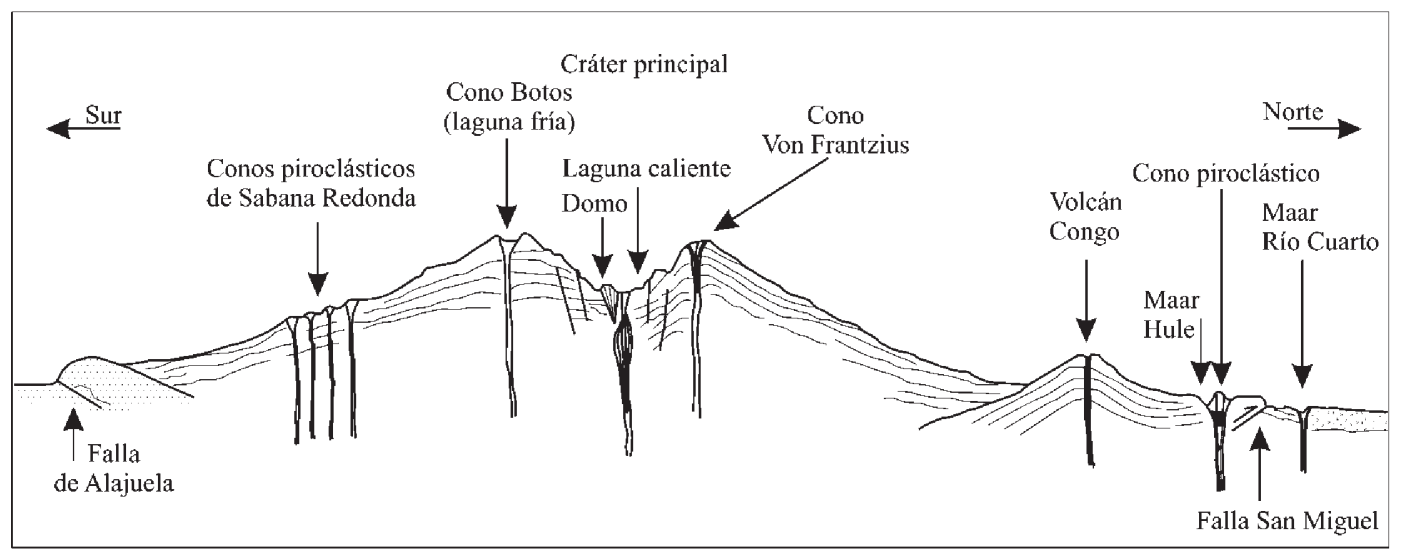

Fig. 2: Corte transversal esquemático del volcán Poás y alrededores (sin escala). Modificado de Soto \& Alvarado (1989). 
Soto (1999) incluye en la Subunidad Congo Inferior el grueso de los depósitos proximales del Congo, mayormente basaltos porfiríticos (plag $+\mathrm{ol} \pm \mathrm{px}$ ) con un porcentaje de sílice entre 49 y 53\% (Tournon, 1984; Malavassi, 1991) y dentro de la Subunidad Congo Superior agrupa las lavas que afloran en la parte nororiental del Congo y que se extienden hasta el maar de Hule, sitio en donde fueron destruidas en parte por la génesis de este maar. Son en general andesitas basálticas, algunas porfiríticas, algunas afíricas.

La Subunidad Distal (Soto, 1999) está compuesta por epiclastos y piroclastos de flujo. En los ríos María Aguilar y Sardinal aflora un espeso depósito de lodo (Rojas, 1993), que contiene numerosas bombas de basaltos afíricos vítreos, de tamaño centimétrico a métrico, interpretados como un enorme lahar originado durante la evacuación explosiva de las bombas contenidas. En parte es cubierto por lavas de composición similar de la Subunidad Congo Inferior. Las partes más distales de esta subunidad, en el sector norte del maar de Hule, son básicamente lahares y otros epiclastos provenientes del volcán Congo.

\section{Depresión de Bosque Alegre}

Entre el volcán Cerro Congo y el Escarpe de San Miguel se encuentra la depresión de Bosque Alegre, que aloja a las lagunas Hule y Congo y una menor innominada, así como una loma de origen volcánico (Fig. 2). Es de forma ovalada en planta, con un eje mayor de 2,25 km y el menor de $1,75 \mathrm{~km}$, para un área de $4 \mathrm{~km}^{2}$. Comprende los productos emitidos desde el maar de Hule y el cráter de explosión Pata de Gallo. Sobreyacen localmente a los productos del volcán Cerro Congo. Son básicamente piroclastitas de oleadas, de flujo, de caída, una lava intramaárica y un cono de piroclastos intramaárico. La lava y los depósitos del cono intramaáricos son basaltos con olivino (Tournon, 1984; Prosser \& Carr, 1987; Malavassi, 1991). Estos depósitos son incluidos bajo la denominación de Unidad Bosque Alegre (Soto, 1999)

Los depósitos de explosión del maar de Hule son el resultado de tres fases principales. La primera fase hidromagmática se caracteriza por contener más clastos líticos que la tercera $(\sim 45 \%$ contra $20 \%$ ). Una fase intermedia es freática. En cortes más distales, al lado occidental del maar, sin embargo, solo se reconocen las dos fases últimas (Soto, 1990; 1999). En este sector del maar aparece además una capa de ceniza fina de caída, correspondiente con la nube de ceniza producida por las erupciones. El volumen producido por el maar de Hule fue de $\sim 2 \mathrm{~km}^{3}$ de piroclastos (Soto, 1999).

Con base en la edad que Melson et al. (1988) obtuvieron para el último evento del Congo, que antecede a la formación del maar de $\mathrm{Hu}$ le, los autores estimaron que éste debe haberse formado hace unos 3000 años. Al calibrar una edad de radiocarbono publicada por Malavassi et al. (1990), se obtiene una edad de 2790+70-20 años a.P. y se considera que esta edad de $\sim 2800$ años está acorde con el evento maárico de Hule. Por otra parte, las edades calibradas aportadas por Horn (2001) determinadas sobre maderas y hojas en piroclastitas de Hule se encuentran entre 3330 y 2180 años a.P (Cuadro 1).

\section{Volcán Barva}

El macizo volcánico del Barva es un complejo andesítico con varios centros eruptivos en la cima y varios conos parásitos en los flancos. En los afloramientos septentrionales del Barva se encuentran asociadas múltiples estructuras volcánicas y erosivas. En la parte cuspidal hay varias calderas anidadas bien identificadas (Alvarado, 1989; Soto, 1994; Alvarado, 2000). En el pie noroccidental de la estructura norte se encuentra el cono La Legua (Soto, 1999), uno de los conos pericaldéricos que presenta morfología joven y lavas que se disponen con un patrón radial. Otra estructura volcánica está representada por el cráter Río Volcán, que es un cono doble con un delgado anillo de tobas alrededor (Alvarado, 1989), cuyo origen se atribuye a explosiones freatomagmáticas.

Adosado al flanco septentrional descendente del Barva (Soto, 1999), se encuentra el cono Río Peje, que pareciera ser piroclástico, luego el cono Pozo Azul, que se distingue como un cono que semeja un centro de emisión en forma de escudo, desde el cual se desarrolla un patrón de drenaje semirradial hacia el norte, con morfología joven. 
Cuadro 1

Dataciones de radiocarbono de Hule y Congo y sedimentos de las lagunas Hule y María Aguilar

\begin{tabular}{|c|c|c|c|c|c|}
\hline Referencia & Muestras y contexto & Edad a.P & $\begin{array}{c}\text { Edad } \\
\text { calibrada a.P. }\end{array}$ & $\begin{array}{l}\text { Edad calibrada } \\
\qquad(1 \sigma) . \text { a.P. }\end{array}$ & $\begin{array}{c}\text { Rango de } \\
\text { edad calibrada } \\
(2 \sigma) \text { a.P. }\end{array}$ \\
\hline Melson et al. 1988 & $\begin{array}{l}\text { Tronco en flujo } \\
\text { piroclástico del Congo }\end{array}$ & $5140 \pm 110$ & 5910 & $5990-5750$ & $6170-5620$ \\
\hline Soto (1999) & $\begin{array}{l}\text { Tronco en flujo } \\
\text { piroclástico del Congo }\end{array}$ & $5330 \pm 130$ & $\begin{array}{l}6170,6140, \\
6110,6070 \\
6060,6010 \\
6000\end{array}$ & $6280-5930$ & $6400-5760$ \\
\hline $\begin{array}{l}\text { Malavassi et al. } \\
\text { (1990) }\end{array}$ & $\begin{array}{l}\text { Tronco en tefras } \\
\text { del Congo }\end{array}$ & $2730 \pm 50$ & $\begin{array}{l}2841-2828 \\
2787\end{array}$ & $2870-5930$ & $2050-2750$ \\
\hline Horn (2001) & $\begin{array}{l}\text { Madera a 2,66 m } \\
\text { en el núcleo } 3 \text { de Hule }\end{array}$ & $\begin{array}{l}3080 \pm 70 \\
3270\end{array}$ & 3330,3280 & $3380-3170$ & $3450-3080$ \\
\hline Horn (2001) & $\begin{array}{l}\text { Hoja a } 1,45 \mathrm{~m} \\
\text { en el núcleo } 3 \text { de Hule }\end{array}$ & $1250 \pm 60$ & 1180 & $1260-1070$ & $1290-1000$ \\
\hline Horn (2001) & $\begin{array}{l}\text { Hoja a } 2,94 \mathrm{~m} \\
\text { en el núcleo } 3 \text { de Hule }\end{array}$ & $\begin{array}{l}2230 \pm 60 \\
2210,2190 \\
2180\end{array}$ & 2310,2240 & $2340-2150$ & $2350-2070$ \\
\hline Horn (2001) & $\begin{array}{l}\text { Hoja sobre ceniza } \\
\text { en María Aguilar (núcleo 1) }\end{array}$ & $2520 \pm 30$ & $\begin{array}{l}2712,2625 \\
2623\end{array}$ & $2736-2496$ & $2744-2471$ \\
\hline Horn (2001) & $\begin{array}{l}\text { Fragmento de madera } \\
\text { (núcleo 3) de María Aguilar }\end{array}$ & $2610 \pm 70$ & 2750 & $2780-2730$ & $2850-2490$ \\
\hline
\end{tabular}

\section{TEFROESTRATIGRAFÍA}

Se levantaron unas 70 secciones estratigráficas de las tefras en el área delimitada por los cursos de los ríos Toro y Sarapiquí y en una transecta más al norte en el camino que une las poblaciones de Río Cuarto y San Miguel. Se describieron perfiles distribuidos a lo largo de los cursos mencionados en ambas márgenes del río Toro, desde Río Cuarto hasta Bajos del Toro; en el río Sarapiquí desde San Miguel hasta Cinchona y en las inmediaciones de la laguna Hule desde Colonia del Toro hasta el poblado de Los Ángeles (Figs. 3, 4, 5, 6 y 7). Como base topográfica se utilizaron las hojas Poás, Quesada y Río Cuarto a escala 1: 50000 del Instituto Geográfico Nacional. Los datos colectados se han vertido en mapas de isopacas y de isopletas (con juveniles y no juveniles) con el fin de analizar las variaciones de espesor y granulometría de estos depósitos e identificar las fuentes de procedencia (Figs. 8, 9 y 10).

\section{Tefras antiguas}

Los depósitos inferiores pueden llegar a alcanzar espesores anómalamente grandes (hasta $8 \mathrm{~m}$ ). Corresponden con lapillitas y tobas lapillíticas café anaranjadas oscuras, muy alteradas, masivas, con fragmentos líticos no juveniles totalmente oxidados. Los fragmentos juveniles tienen un $\Phi_{\text {Max }}=1-2 \mathrm{~cm}$, en algunos depósitos llegan a $4 \mathrm{~cm}$ y presentan un $\Phi_{\text {Max }}=0,5 \mathrm{~cm}$ hasta 2 $\mathrm{cm}$. Los juveniles (> 85\%) suelen ser pómez poco vesiculadas y espinosas a poco vesiculadas (juveniles angulares), grises (sanas), anaranjadas (alteradas), sugiriendo un vulcanismo freatomagmático. En el área de Isla Bonita se pueden diferenciar una serie de al menos 4 unidades de $2 \mathrm{~m}$ de espesor separadas por suelos de 20 a 30 $\mathrm{cm}$. Son portadoras de bloques de $13 \times 8 \times 6 \mathrm{~cm}$ y no juveniles de hasta $9 \mathrm{~cm}$. Suelen presentar niveles de óxidos de hierro y manganeso. El contacto entre granos es puntual; el grado de coherencia del depósito es variable y la selección 


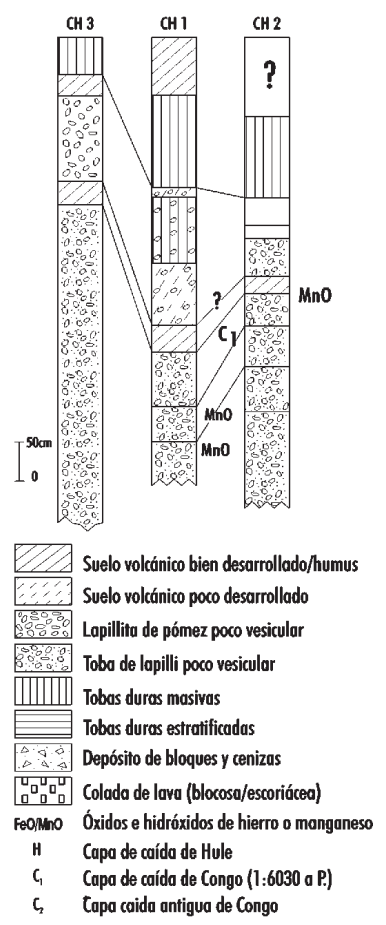

Fig. 3: Secciones estratigráficas levantadas en el área de Santo Domingo, río San Rafael y BM1756. Predominio de depósitos lapillíticos de tefras antiguas. buena. Hacia arriba los depósitos lapillíticos pierden los rasgos piroclásticos primarios y se transforman en un suelo volcánico.

Entre los poblados de Cariblanco y Ujarraz, se observa que estos depósitos plinianos subyacen claramente a las lavas del Von Frantzius, presumiblemente de una edad $\geq 40000$ años, por lo que estas tefras serían más antiguas en su mayoría, si no todas. Sin embargo, cerca del Poás (fuera del área de estudio), hay tefras datadas en 33 000 años y menos (datos inéditos de G.E. Alvarado), algunas procedentes de Poás y otras del Barva-Guararí.

La correlación estratigráfica de estos depósitos resultó extremadamente difícil llevarla a cabo más allá de algunos kilómetros de separación entre columnas, en la mayoría de los casos, por la inexistencia de capas guías claras y continuas, así como la ausencia de paleosuelos datables, pobres en materia orgánica. Por otra parte, la granulometría varía extrañamente muy poco con los depósitos (usualmente entre 2 y $3 \mathrm{~cm}$ de diámetro por varias decenas de kilómetros), aunada a la inexistencia de líticos no juveniles de mayor tamaño de grano, así como de capas con otra composición petrográfica (basálticas por
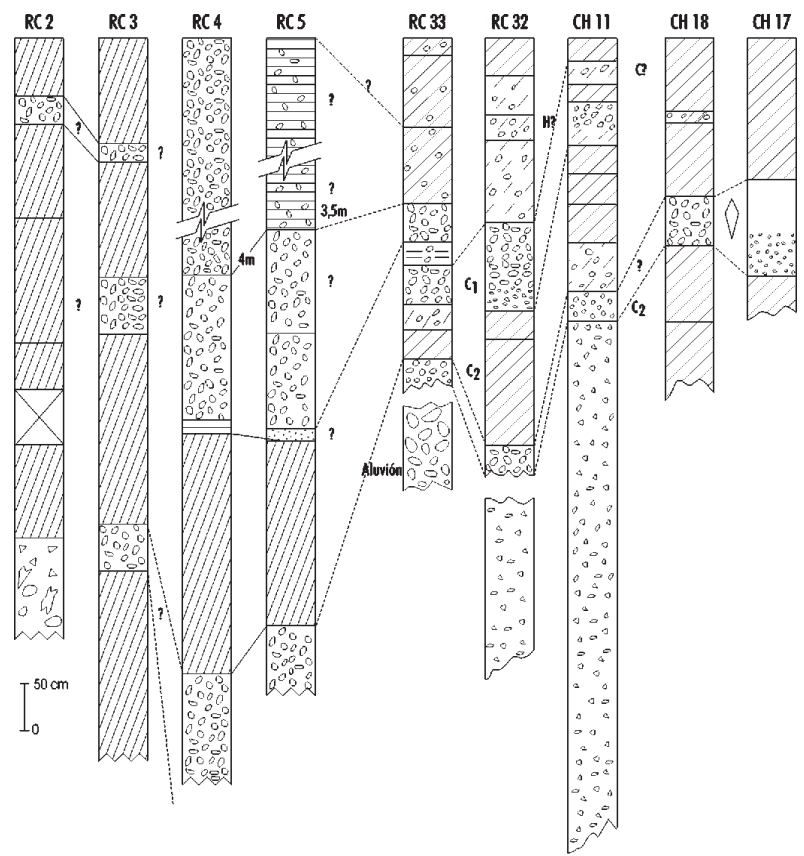

Fig. 4: Secciones en el río Sardinal, río Hule, río Tercero, Río Cuarto y San Miguel. En RC2 se observa el lahar relacionado con el volcán Congo. La simbología es la misma que en la figura 3. 


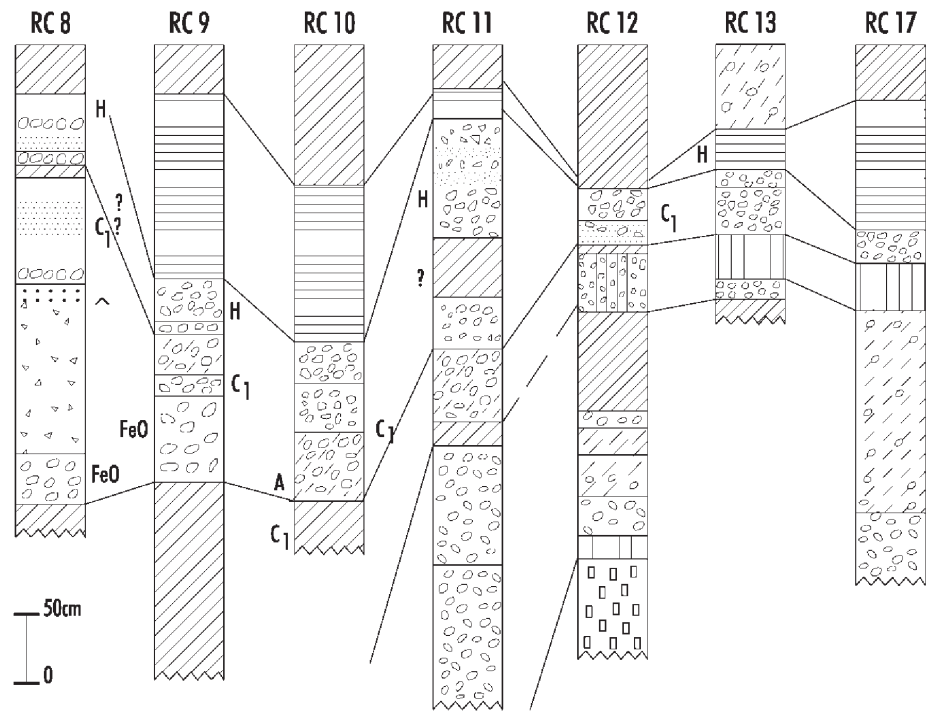

Fig. 5: Tefras de Congo, Hule y tobas estratificadas asociadas con Hule en quebradas Honda, Palmera, Mica, La Gata y río Pozo Azul. La simbología es la misma que en la figura 3.

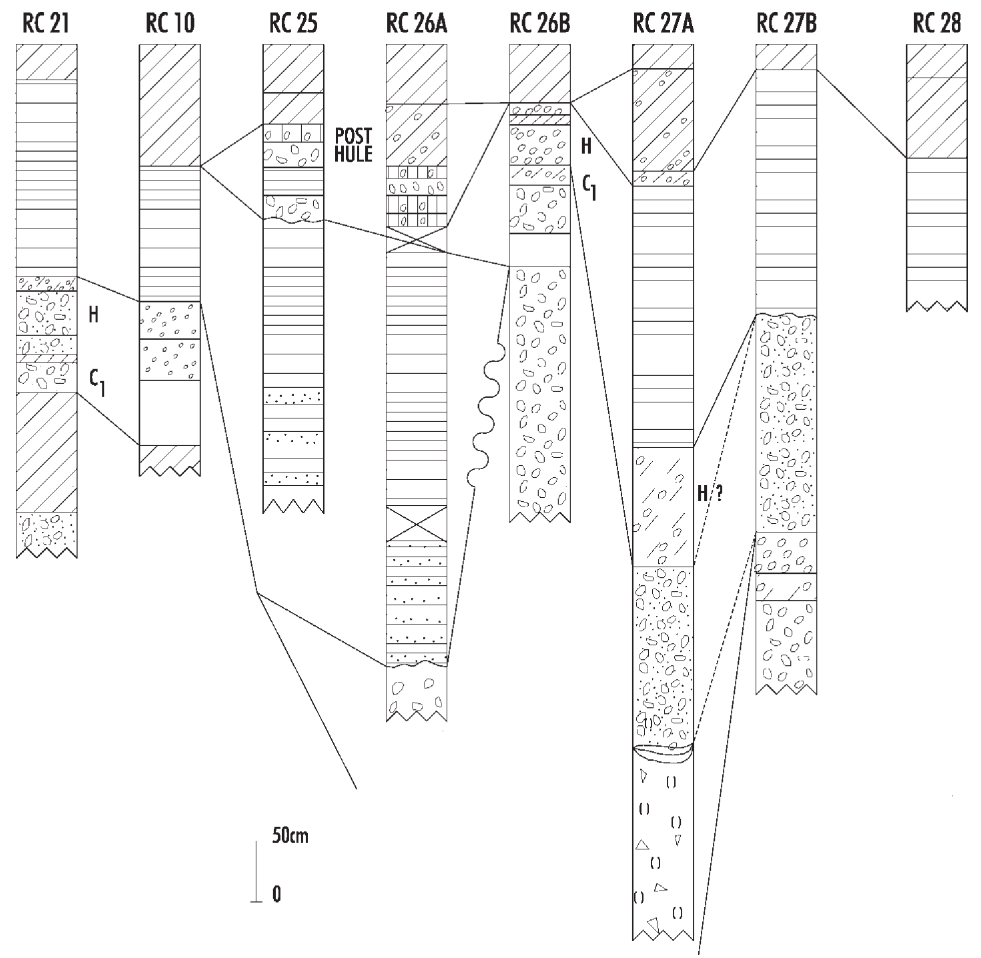

Fig. 6: Depósitos de caída de Congo y Hule. En las secciones RC27 A y B se observan los flujos piroclásticos de Congo y en RC25 la facies post- Hule. La simbología es la misma que en la figura 3. 


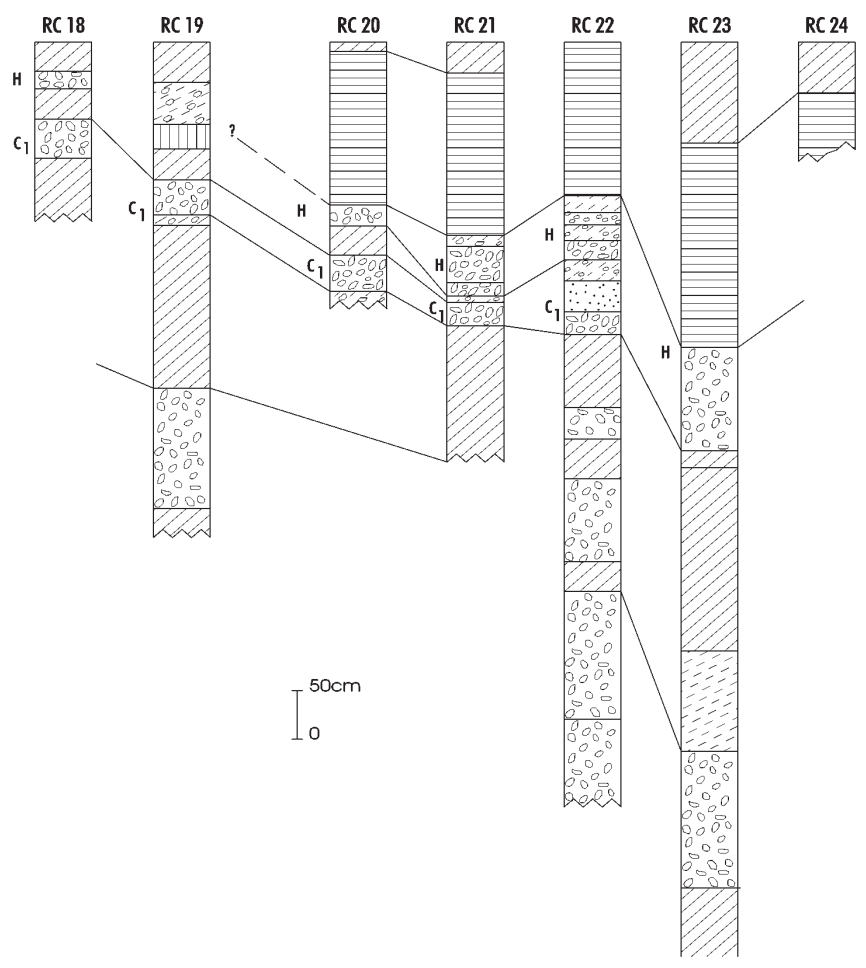

Fig. 7: Depósitos de caída de Congo y Hule y facies de tobas estratificadas de Hule cubriendo a las anteriores. La simbología es la misma que en la figura 3.

ejemplo), lo cual, en su conjunto, dificulta la correlación y el establecimiento de mapas de isopletas e isopacas. Así, no solo se presentaron problemas de correlación, sino que en cortas distancias, los espesores variaban considerablemente. Lo anterior se explica parcialmente por la presencia de flujos pumíticos en la menor cantidad de los casos (CH 11; Fig. 4) y en su mayoría por existir profundos niveles de erosión de todos estos depósitos, poniendo cerca de la superficie a depósitos de distintas edades y, por ende, con posición estratigráfica diferente. Esto explica por qué el tamaño del grano varía muy poco en decenas de kilómetros y los espesores tan contrastantes para depósitos en su mayoría de caída. De este modo, pese a que se trató de determinar la fuente, podría establecerse que son varias, probablemente los volcanes Barva y Poás y otros centros menores al norte del Barva.

Sobreyaciendo a los depósitos descritos en la región de Río Cuarto-San Miguel, se presentan tobas consolidadas de colores verdosos, ricas en cristales. Muestran un espesor máximo de $2 \mathrm{~m}$ en la sección de Santo Domingo y $1 \mathrm{~m}$ en la quebrada de Piedra Grande y disminuye súbitamente en la sección CH 3 (BM 1756; Fig. 3) donde tiene un espesor de $30 \mathrm{~cm}$. Son depósitos con laminación paralela e inclinada, portadoras de materia orgánica y troncos con reemplazo parcial por sílice. En otros perfiles la estratificación es difusa y está definida por alternancia de granulometrías finas y más gruesas. Cuando están alteradas el grado de consolidación disminuye y presentan un aspecto más suave. Posiblemente son depósitos atribuibles a oleadas piroclásticas.

\section{Depósitos asociados con el Volcán Congo}

\section{Flujo piroclástico}

Flujos de pómez densas a vesiculadas, cubren a prácticamente todas las unidades del Congo. 
Los trabajos anteriores los atribuyen al Congo, aunque se requiere de mayores estudios. También se distingue al menos un depósito de pómez de caída asociado. Este se encuentra a $10 \mathrm{~km}$ al norte del volcán, $\sim 1 \mathrm{~km}$ al SE del poblado de Río Cuarto, donde aparece masivo con $35 \mathrm{~cm}$ de espesor y pómez tan grandes como $6 \mathrm{~cm}$. En los alrededores de la laguna Hule, los sobreyace una ceniza morada, que a su vez subyace a los depósitos hidromagmáticos de Hule, como guías coherentes. Con base en las características texturales de las pómez, se infiere que hubo al menos una erupción pliniana, con flujos de bloques y cenizas contemporáneos, quizás por la destrucción de un domo. Su composición química es la de una andesita ácida con mediano a bajo contenido de potasio (Soto, 1999).

Los flujos de pómez han sido identificados tan lejos como la quebrada Palmera (255,55 N - 510,15 E; RC8 Fig. 5), a 7 km del cráter. Forman un manto de hasta varios metros de espesor. La edad calibrada de los flujos pumíceos es de $6030+250$ - 300 años a.P (Soto, 1999). En las inmediaciones de quebrada Honda aflora con un espesor de $2 \mathrm{~m}$ y en la quebrada Berros presenta un espesor $>3 \mathrm{~m}$, sin observarse la base. En quebrada Honda (Fig. 6) se presenta como un depósito pardo con fragmentos juveniles de color blanco, subredondeados y fragmentos grises juveniles con un $\Phi_{\text {Max }}$ de $4 \mathrm{~cm}$ flotantes en una matriz café clara. En la quebrada Culebra también se observaron depósitos pumíceos, posiblemente de flujo.

En la quebrada Berros (RC 27A y B; Fig. 6), el flujo piroclástico muestra dos secciones separadas por una ceniza mediana a gruesa de $4 \mathrm{~cm}$ de espesor que presenta niveles lenticulares, estructuras de truncamiento y adelgazamientos laterales. La sección superior, de color gris claro a violácea, está alterada en un primer tramo de $1 \mathrm{~m}$ para continuar con 1,5 m del depósito color pardo con juveniles de $\Phi_{\text {Max }}=3 \mathrm{~cm}$ y no juveniles de 3-4 cm, en cuya base se destacan líticos de $8 \mathrm{~cm}$. Hacia la parte media del depósito los juveniles tienen un $\Phi=2-3 \mathrm{~cm}$. La sección inferior muestra menor proporción de juveniles, granulometría más fina y lapilli acrecional de $1 \mathrm{a} 3 \mathrm{~cm}$. Soto (1990) describe depósitos pumíceos similares en un sector más occidental del volcán Congo en la quebrada La Higuera y ríos Pozo Azul y Seco, pero con pómez y líticos de mayor granulometría (10-12 cm) y espesores del orden de los $3 \mathrm{~m}$, aunque tampoco observa la base.

\section{Lahar}

Los afloramientos se restringen a la sección RC2 (Fig. 4) en el área de Río Tercero. Corresponde a un depósito caótico de ceniza retrabajada, arcillosa de color gris oscuro, con cantos rodados, enriquecido en grandes troncos. La madera está quemada totalmente o presenta bordes de deshidratación. El depósito posee un espesor mínimo de $1,5 \mathrm{~m}$. Se distinguen fragmentos de dacitas, redondeados, con $\Phi_{\text {Max }}=1,5 \mathrm{~cm}$ y fragmentos juveniles vesiculados de $\Phi=15 \mathrm{~cm}$, de color gris oscuro. En los ríos Sardinal, María Aguilar, Hule y Río Tercero (cursos medios y superiores) depósitos de esta naturaleza fueron reconocidos por Rojas (1993) y por Arroyo \& Alvarado (2002). Una datación radiocarbónica (Cuadro 2) demuestra la contemporaneidad entre este depósito lahárico $(5110 \pm 80$ años, calibrado en 5905+25-160 a.P., con base en Stuiver et al., 1998), en correspondencia con la edad del flujo piroclástico previamente descrito, cuya edad calibrada es $6030+250-300$ años a.P.

\section{Tefras de caída}

Los depósitos de tefra de caída se relacionan presumiblemente con el volcán Congo, dado que son dacitas con anfíbol, composición característica de las emisiones de este centro. Se han reconocido en la falda norte del volcán, al noroeste de la laguna Hule y en el tramo inferior del río Toro (RC8, RC9, RC10, RC12, RC18, RC19, RC20, RC21; Figs. 5 y 7). En general están separados de las unidades más antiguas por suelos y sobreyacidos por los depósitos de tefras asociados al evento de Hule y separados por un suelo de mucho menor desarrollo (Fig. 5). El espesor de los depósitos varía en un rango entre $80 \mathrm{~cm}$ y $20-15 \mathrm{~cm}$ en el sector más occidental del volcán Congo, en la margen izquierda del río Toro (Fig. 8). El color es pardo claro, se presentan 
Cuadro 2

Dataciones de radiocarbono provenientes de perforaciones y de un depósito lahárico relacionado con el volcán Congo, realizadas para el P.H. Cariblanco (Alvarado y Salani, 2002)

\begin{tabular}{|c|c|c|c|c|c|}
\hline Número de muestra & Localidad perforación & Ubicación & Material & Profundidad $(\mathrm{m})$ & Edad (años) \\
\hline $\begin{array}{l}\text { P.H. Hule } \\
\text { P.14 (GX- } \\
25765)\end{array}$ & PHL-14 & $\begin{array}{l}248347- \\
517857\end{array}$ & $\begin{array}{l}\text { Arenas volcánicas } \\
\text { finas con materia } \\
\text { orgánica }\end{array}$ & $\begin{array}{l}6,30-8,7 \\
(8,3)\end{array}$ & $>42400$ \\
\hline $\begin{array}{l}\text { PHC 26/33 } \\
\text { (GX-25768) }\end{array}$ & PHC-26 & $\begin{array}{l}252500- \\
515282\end{array}$ & $\begin{array}{l}\text { Toba de lapilli con } \\
\text { materia orgánica y } \\
\text { cenizas. }\end{array}$ & $\begin{array}{l}33,30-34- \\
40 \mathrm{~m}\end{array}$ & $>42680$ \\
\hline $\begin{array}{l}\text { PHC 26/45 } \\
\text { (GX-25767) }\end{array}$ & PHC-26 & $\begin{array}{l}252500- \\
515282\end{array}$ & $\begin{array}{l}\text { Toba de lapilli } \\
\text { blancuzca (alterada) } \\
\text { con materia orgánica }\end{array}$ & $45,5 \mathrm{~m}$ & $>36420$ \\
\hline $\begin{array}{l}\text { PHC 26/77 } \\
\text { (GX-25766) }\end{array}$ & PHC-26 & $\begin{array}{l}252500- \\
515282\end{array}$ & $\begin{array}{l}\text { Brecha polimíctica } \\
\text { y tobas brechosas } \\
\text { caolinitizadas }\end{array}$ & $77 \mathrm{~m}$ & $>39163$ \\
\hline $\begin{array}{l}\text { SPQYAL } \\
(\text { GX-25819) }\end{array}$ & $\begin{array}{l}\text { S. Presa } \\
\text { Quicuyal }\end{array}$ & $\begin{array}{l}249450- \\
515900\end{array}$ & $\begin{array}{l}\text { Madera dentro de } \\
\text { una brecha volcánica } \\
\text { (flujo piroclástico?) }\end{array}$ & $20 \mathrm{~m}$ & $>43780$ \\
\hline $\begin{array}{l}\text { RC 2-1 } \\
\text { (GX-28644) }\end{array}$ & $\mathrm{RC}-2$ & $\begin{array}{l}257400- \\
513300\end{array}$ & Lahar caliente & $\sim 5 \mathrm{~m}$ & $5110 \pm 80$ \\
\hline
\end{tabular}

con buena selección y granosoportado. Están representados por capas de lapilli y tobas de lapi1li. En los sectores distales, como Río Cuarto (RC 19, 20, 21 y 22; Fig. 7) están compuestos por juveniles grises, pómez blancas con $\Phi_{\mathrm{Max}}=$ 1-2 cm. En la base aparecen fragmentos aislados morados y blancos $<5 \mathrm{~cm}$ y juveniles dacíticos de $5 \mathrm{~cm}$. En quebrada Honda (RC9) son lapillitas con fragmentos juveniles dacíticos con anfíboles de $3 \mathrm{~cm}$ y no juveniles de hasta $6 \mathrm{~cm}$. En las facies más proximales, en quebrada Mica (RC10), presentan pómez de $\Phi_{\text {Max }}=4-5 \mathrm{~cm}$ con aislados líticos juveniles poco vesiculados de 12 $\mathrm{cm} \times 10 \mathrm{~cm}$ x $7 \mathrm{~cm}$, no juveniles de hasta $20 \mathrm{~cm}$ y líticos morados de hasta $7 \mathrm{~cm}$. Las características del depósito piroclástico estarían indicando un origen por caída.

A estos depósitos de caída del Congo se les ha llamado $\mathrm{C}_{1}$ para diferenciarlos de otros depósitos pumíceos (pómez de caída con anfíbol) más antiguos observados en las columnas RC32

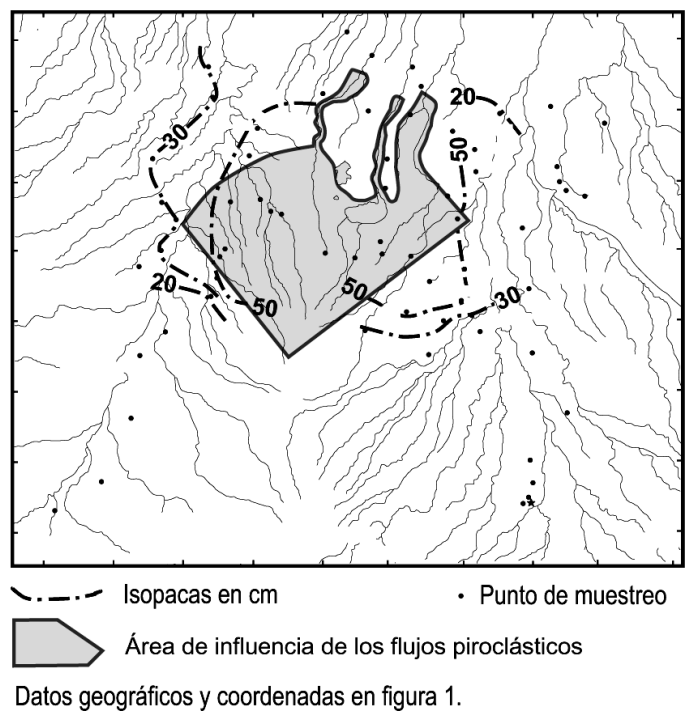

Fig. 8: Mapa de isopacas de tefras de caída del volcán Congo (6030 a.P.) y área de influencia mínima de flujos piroclásticos. 
y RC33, CH11, 17 y 18 (Fig. 4), presumiblemente relacionados con una erupción previa del Congo (llamada $\mathrm{C}_{2}$ ). La $\mathrm{C}_{2}$ es una capa de pómez con anfíbol de entre 30 y $50 \mathrm{~cm}$ de espesor con pómez de hasta $6 \mathrm{~cm}$ de diámetro. Sin embargo, con base en la distribución de las isopacas (Fig. 8), también puede interpretarse como proveniente de la laguna Hule. De demostrarse esto, Hule resultaría ser un maar policíclico o resurgente.

\section{Maar Hule}

Dentro del evento explosivo relacionado con el centro volcánico Hule, se encuentra una facies gruesa y una fina atribuidas a caída y una facies vinculada a procesos de oleadas piroclásticas. En general muestran una buena correlación a lo largo de diferentes secciones. De acuerdo con varias fuentes (Cuadro 1) se estima una edad de 2800 años para los productos de las fases piroclásticas principales de este centro emisor. Por último, se ha reconocido una facies explosiva posterior a las emisiones principales del maar de Hule que aflora en el sector occidental de la laguna homónima.

\section{Facies de caída gruesa}

La facies gruesa tiene sus mejores exposiciones en el camino que une Río Cuarto con San Miguel. Está representada por depósitos masivos sin estratificación, de color gris blanquecino, granosoportados, con un espesor que varía (1,7 a $5 \mathrm{~m}$ ), compuestos por un predominio de lapilli juveniles sanos y líticos con distinto grado de alteración, oxidados e hidrotermalizados. Los juveniles sanos son densos de color gris verdoso oscuro con un $\Phi_{\text {Max }}=4 \mathrm{~cm}$ y $\Phi_{1 / 2}=1-2 \mathrm{~cm}$; los fragmentos líticos no juveniles se presentan de color blanco verdoso cuando están completamente alterados, y rojizos cuando están oxidados e hidrotermalizados. Poseen un $\Phi_{\text {Max }}$ de 7-10 cm (Fig. 9). En la base de los depósitos aparecen capas de lapilli más gruesos con $\Phi_{1 / 2}=1-4 \mathrm{~cm}$, en los que se destacan bloques de caída no juveniles de $8 \mathrm{~cm}$ de longitud y juveniles del mismo tamaño. De acuerdo con los atributos texturales del depósito, se le atribuye una génesis de caída.

\section{Facies de caída fina}

Esta facies está representada por capas de ceniza morada y de lapilli café claro con un espesor que varía desde 10 y $40 \mathrm{~cm}$. Se reconoce en las quebradas Flor, Honda y Palmera (RC8, RC21 y RC22; Figs. 1 y 7). Las capas de lapilli suelen presentar estratificación difusa y gradación en rombo. El depósito está integrado mayormente por juveniles con $\Phi_{1 / 2}$ homogéneos y en el orden de los 1-2 cm y, excepcionalmente, de 3 y $5 \mathrm{~cm}$ (Fig. 9). Presenta un carácter granosoportado. Infrayace a las tobas estratificadas de Hule. Los rasgos texturales del depósito indican un origen por procesos de caída.



Fig.9: Mapa de isopletas líticos e isopacas de las tefras de caída del maar Hule (2800 a.P).

\section{Tobas estratificadas}

Cubren un área amplia que se extiende desde la laguna Hule hasta $7 \mathrm{~km}$ al oeste del centro emisor y unos $3 \mathrm{~km}$ al suroeste (Figs. 5, 6 y 7). Presentan una asimetría en los espesores con un máximo localizado en las inmediaciones de la laguna y el segundo en el área occidental en el sector de quebrada Honda y a $1 \mathrm{~km}$ de la casa de máquinas de Toro (Fig. 10). Los espesores medidos están en un rango en general de 1 a $2,5 \mathrm{~m}$ y un mínimo de $35 \mathrm{~cm}$ en las secciones más alejadas sobre el río Toro. Corresponden con 
una secuencia de capas de lapilli grueso a medio alternadas con tobas lapillíticas estratificadas duras, de color beige a café oscuro, con estratificación centimétrica a decimétrica, estructura planar interna horizontal integrada por lapilli de $\Phi_{1 / 2}=1$ $\mathrm{cm}$. En el sector cercano a la casa de máquinas de Toro II, la toba se encuentra rellenando canales (RC13). En las proximidades de la laguna Hule, los depósitos muestran mayor granulometría y se reconocen clastos basálticos de proyección balística de hasta $80 \times 50 \times 40 \mathrm{~cm}$.

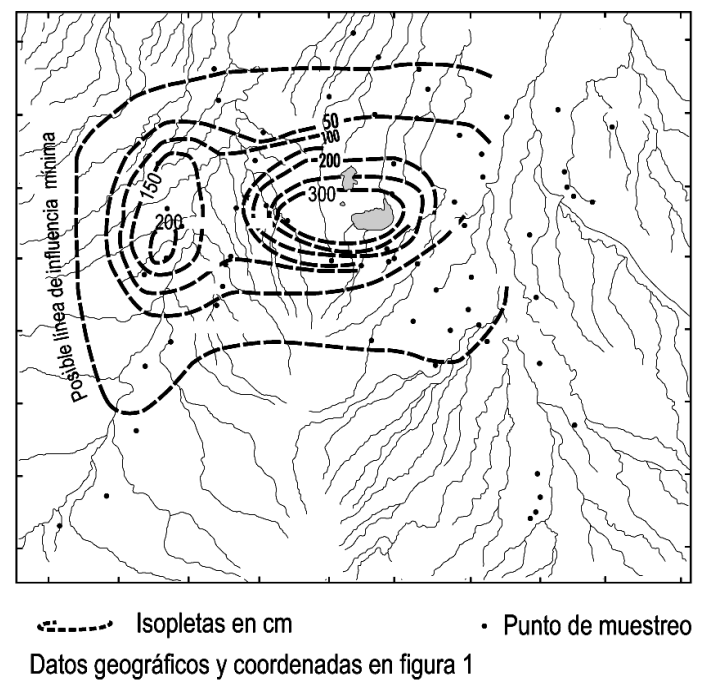

Fig. 10: Mapa de isopletas de oleadas piroclásticas superiores de Hule (2800 a.P).

Las características texturales del depósito permiten atribuirle un origen por oleadas piroclásticas. Los dos máximos en los espesores encontrados (Fig. 10) podían ser en el primer caso y más cercano, el resultado de oleadas piroclásticas basales (base surge) y en el otro caso (segundo máximo) el resultado de una componente de caída asociada con la oleada (ash cloud surge). Los depósitos cubren a las tefras de caída relacionadas con el centro Hule.

\section{Facies explosiva Post- Hule}

Los productos de este evento han sido reconocidos en quebrada Honda y a 1200 m de la entrada a Colonia Toro (RC25; Fig. 6), donde cubren tobas estratificadas atribuidas a Hule. El contacto es transicional o pobremente definido. Poseen un espesor entre 28 a $40 \mathrm{~cm}$ y corresponden con tobas duras con trazas de lapilli y lapillitas, estratificación difusa y granulometrías promedio de $2 \mathrm{~cm}$. Con base en dataciones relativas de Horn (2001), obtenidas a través de tasas de sedimentación, se asume que un evento asociado con Hule ocurrió unos 1700 años a.P. y otro unos 670 años a.P. Sin embargo, el estudio no presenta descripciones de los depósitos extraídos de los núcleos de los sedimentos lacustres de Hule y alrededores para una mejor correlación.

\section{CONCLUSIONES}

Los estudios tefroestratigráficos han permitido distinguir en el sector norte de los macizos del Poás y Barva, diferentes unidades explosivas correspondientes con varios eventos.

Los depósitos más antiguos son depósitos de caída y de flujo de gran espesor y moderado a alto grado de meteorización, que se encuentran intercalados con tobas estratificadas y masivas. Estas unidades están sobreyaciendo a las lavas antiguas del PaleoBarva y del PaleoPoás y están cubiertas parcialmente por las lavas del Von Frantzius ( 40 000 años) y por los depósitos holocénicos relacionados con las dos últimas erupciones del volcán Congo (facies de caída, de flujo piroclástico y lahares), las más recientes de Poás y las de Hule (últimos 2800 años). Son tobas y tobas de lapilli, con $85 \%$ de pómez, cuyos rasgos texturales indican un origen freatomagmático. Se reconocen por lo menos 4 unidades separadas por suelos. El análisis estratigráfico sugiere que los depósitos proceden de distintas fuentes, las más probables Barva, Poás y otros centros menores al norte del Barva.

Asociados con el volcán Congo, se identificaron depósitos de flujo piroclástico, lahares y capas de caída. Los flujos piroclásticos del volcán Congo son los de mayor importancia dentro del contexto del presente estudio. Son flujos de pómez densas a vesiculadas que sobreyacen a prácticamente todas las unidades del 
Congo. Estos depósitos, datados en 6030 años, tienen espesores sin base visible de 2-3 m. Hubo al menos un episodio que produjo flujos de bloques y cenizas contemporáneos con una erupción pliniana de este evento.

En el área de Río Tercero se reconoció un lahar, con una edad y fuente de origen congruentes con la del flujo del Congo. Es un depósito caótico compuesto por material volcánico fino retrabajado, con fragmentos de dacita.

La facies de caída atribuida a este centro, en general está separada de las unidades más antiguas por suelos y cubierta por los depósitos de tefras asociados al evento de Hule. Son capas de tobas y tobas de lapilli con juveniles de andesitas y dacitas con anfíboles. Se identificó una capa de pómez con características petrográficas similares $\left(\mathrm{C}_{2}\right)$ que provendría de una erupción más antigua del Congo o podría también relacionarse con el maar de la Laguna Hule.

Vinculados con el maar de Hule se reconocen depósitos de caída y de oleadas de naturaleza freatomagmáticos. Entre la facies de caída se distinguen por una parte depósitos masivos de lapillitas con líticos alterados e hidrotermalizados, con capas de lapilli más gruesos en los sectores basales. Por otra parte, se distinguen capas de granulometría más finas, de ceniza, de color morado y de lapilli café claro, con estratificación difusa y gradación en rombo. Los depósitos de oleadas piroclásticas son lapillitas gruesas a medianas y tobas lapillíticas estratificadas, que sobreyacen a las tefras de caída. En parte son oleadas piroclásticas basales y en parte a oleadas piroclásticas asociadas con nubes de ceniza, acompañadas con una componente de caída.

Cubriendo a la facies de tobas y lapillitas de 2800 años de Hule, y como último registro de las efusiones explosivas de este sector, se disponen los productos de la facies que denominamos post-Hule. Corresponden con tobas duras con trazas de lapilli y lapillitas. El contacto transicional o pobremente definido con los depósitos anteriores podría indicar una continuidad del evento de Hule y las emisiones corresponderían con periodos eruptivos menores, de 1700 y 670 años a.P.

\section{REFERENCIAS}

ALVARADO, G.E., 1989: Los volcanes de Costa Rica. - 175 págs. Editorial UNED, San José.

ALVARADO, G.E., 2000: Volcanes de Costa Rica: Geología, historia y riqueza natural. - 265 págs. Editorial UNED, San José.

ALVARADO. G.E. \& SALANI, F.M., 2002: Evaluación del peligro y riesgo volcánico para los Proyectos Hidroeléctricos Cariblanco y Toro III. - Instituto Costarricense de Electricidad, 72 págs. [Inf. interno].

ARROYO, I.G \& ALVARADO, G.E., 2002: Evaluación sismotectónica de la falla San Miguel, con énfasis en los alrededores del P.H. Cariblanco. - Instituto Costarricense de Electricidad, 26 págs. [Inf. interno].

HORN, S.P., 2001: The age of the laguna Hule explosion crater, Costa Rica, and the timing of subsequent tephra eruptions: evidence from lake sediments. - Rev. Geol. Amér. Central, 24: 57-66.

MALAVASSI, E., 1991: Magma sources and crustal processes at the terminous of the Central American Volcanic Front. - 435 págs. Univ. de Santa Cruz, California [Tesis de $\mathrm{PhD}$ ].

MALAVASSI R., E., GILL, J.B. \& TRIMBLE, D., 1990: Nuevas dataciones radiométricas del alineamiento volcánico de Poás (Costa Rica): Contribución a la evaluación de peligros volcánicos. - [res.] VII Congr. Geol. de América Central, 19-23 de noviembre de 1990, San José, Costa Rica, Programas y Resúmenes de Ponencias: s.p.

MELSON, W.G., SÁENZ, R., BARQUERO, J. \& FERNÁNDEZ, E., 1988: Edad relativa de las erupciones del Cerro Congo y Laguna Hule. - Bol. Vulcanología, OVSICORI - Universidad Nacional, 19: 8-10.

PROSSER, J.T. \& CARR, M.J., 1987: Poás volcano, Costa Rica: Geology of the summit region and spatial and temporal variations among the most recent lavas. - J. Volcanol. Geotherm. Res. 33: 131-146.

ROJAS, L., 1993: Estudio geológico-geotécnico de un sector del P. H. Laguna Hule. - 43 págs. Escuela Centroamericana de Geología, Univ. de Costa Rica [Campaña geológica].

SOTO, G.J., 1990: Consideraciones volcanológicas y tectónicas preliminares del área de Laguna Hule. Instituto Costarricense de Electricidad, 14 págs. [Inf. interno]. 
SOTO, G.J., 1994: Volcanología física. En: DENYER, P. \& KUSSMAUL, S. (comps.): Atlas geológico Gran Area Metropolitana. - Ed. Tecnológica de Costa Rica: 131-146.

SOTO G.J., 1999: Geología regional de la hoja Poás (1: 50 000). - En: ALVARADO G.E. \& MADRIGAL, L.A. (eds.): Estudio geológico-geotécnico de avance a la factibilidad del P.H. Laguna Hule. - Instituto Costarricense de Electricidad, 15-45 [Inf. interno].

SOTO, G.J. \& ALVARADO, G.E., 1990: Procesos asociados con el agua subterránea. El caso de los volcanes
Arenal y Poás, Costa Rica. - Mem. III Congr. Nac. de Recursos Hídricos, 15-17 de noviembre 1989, 249261; San José.

STUIVER, M., REIMER, P.J., BARD, E, BECK, J.W., BURR, G.S., HUGHEN, K.A., KROMER, B., McCORMAC, F.G., v.d. PLICHT, J. \& SPURK, M., 1998: ${ }^{14} \mathrm{C}$ calibration datasets. - Radiocarbon, 40: 1041-1083.

TOURNON, J., 1984: Magmatismes du Mesozoique a l'actuel en Amerique Centrale. L'example de Costa Rica, des ophiolites aux andesites. - 335 págs. Univ. Pierre et Marie Curie, Paris [Tesis Ph.D]. 